\title{
The role of critical shear stress on acute coronary syndrome
}

\author{
Jongyoun Kim ${ }^{\mathrm{a}}$, Hyemoon Chung ${ }^{\mathrm{a}}$, Minhee $\mathrm{Cho}^{\mathrm{a}}$, Byoung-Kwon Lee ${ }^{\mathrm{a}, *}$, Ali Karimi ${ }^{\mathrm{b}}$ \\ and Sehyun Shin ${ }^{\mathrm{b}, *}$ \\ ${ }^{a}$ Department of Internal Medicine, Yonsei University Medical College, Seoul, Korea \\ ${ }^{\mathrm{b}}$ School of Mechanical Engineering, Korea University, Seoul, Korea
}

\begin{abstract}
Increased aggregation of RBC is associated with many vascular diseases, including acute coronary syndrome (ACS). Critical shear-stress (CSS) as in index of red cell aggregation is defined as either the minimum shear-stress required dispersing the aggregates. The objective of this study is to access the role of CSS in ACS comparing to SA, and to evaluate the correlation with usual biomarkers for atherosclerosis such as fibrinogen, hs-CRP. 169 SA and 223 ACS patients were finally enrolled. A detailed medical history and laboratory data were obtained for each participant from clinical records. CSS is measured by simultaneous measurement of shear stress and light backscattering using a small disposable kit with a microfluidic hemorheometer. We hypothesized that higher value of CSS might be associated increased thrombosis in ACS. As results, relatively younger age was shown and more male in ACS patients, and inflammatory markers (WBC, hs-CRP) were higher in ACS. Whole blood viscosities were significantly higher in ACS than SA along at all shear rates. CSS was $25.7 \%$ higher in ACS (333.8 \pm 147.8$)$ than in SA $(265.4 \pm 149.9 \mathrm{mPa})(p<0.001)$. CSS was highly correlated white blood cell counts, hs-CRP, fibrinogen, and erythrocyte sedimentation rate (ESR). Among those variables, fibrinogen, and ESR were strongly correlated with CSS. We may suggest that CSS could be used as a novel risk marker for ACS.
\end{abstract}

Keywords: Critical shear stress, RBC aggregation, acute coronary syndrome

\section{Introduction}

During the natural evolution of atherosclerotic plaque, an abrupt and catastrophic transition can occur, characterized by plaque disruption, especially when the plaques have much lipid [14]. Atherosclerotic plaque considered prone to disrupt according as plaque vulnerability, or exaggerated hemodynamic condition. Acute coronary syndromes (ACS), including unstable angina (UA) and acute myocardial infarction (AMI), often result from the disruption of a modestly stenotic, unstable, vulnerable plaque [12]. Some patients have a systemic predisposition to plaque disruption that is independent of traditional risk factors. While the traditional risk assessment has been shown to predict long-term outcome in large populations, they fall short in predicting near-future events. Many researchers have tried to identify and to prevent acute coronary syndromes and sudden death [13]. A number of key physiological variables, such

\footnotetext{
*Corresponding author: Byoung-Kwon Lee, Department of Internal Medicine, Gangnam Severance Hospital, Yonsei University, 211 Eonju-ro, Gangnam-gu, Seoul 135-720, Korea. Tel.: +82 2 20193307; Fax: +82 23463 3882; E-mail: cardiobk@ yuhs.ac; Sehyun Shin, School of Mechanical Engineering, Korea University, Anam-dong 5ga, Seongbuk-gu, Seoul 136-713, Korea. Tel.: +82 23290 3377; E-mail: lexerdshin@korea.ac.kr.
} 
as, systolic blood pressure, heart rate, blood viscosity, and multiple endogenous factors exhibit circadian variation and they increased when stressful condition and they may act in concert to produce plaque disruption [9].

Red blood cell (RBC) aggregation is major determinant of whole blood viscosity at a low shear rate. Increased aggregation of RBC is associated with many vascular diseases [3, 10, 19, 20]. To understand $\mathrm{RBC}$ aggregation mechanism and its physiological importance, a variety of indexes have been proposed.

Recently, critical shear stress (CSS) by simultaneous measurement of electrical impedance, capacitance and light backscattering has developed, and is suggested as an index of RBC aggregability [14]. CSS is defined as either the minimum shear-stress required dispersing the aggregates, or the threshold shear required to aggregate RBCs under the shearing hydrodynamic force. The critical shear-stress was determined through a scanning procedure wherein various transient shear stresses were applied. This indicates that a transition should occur from RBCs disaggregation to aggregation [11, 18].

Therefore, we hypothesized that higher value of CSS might be associated increased thrombosis in ACS. The objective of this study is to investigate CSS and other hemorheologic parameters in ACS and to evaluate the correlation with usual biomarkers for atherosclerosis such as fibrinogen, hs-CRP, and ESR.

\section{Materials and methods}

\subsection{Subjects}

Subjects presenting with typical angina who were scheduled coronary angiography at Gangnam Severance hospital between November 2010 and December 2011 were eligible for the study. Patients were diagnosed with stable angina (SA) based on their clinical presentation, ECG, stress test and angiographic findings, with the diagnoses of unstable angina (UA) and acute myocardial infarction (AMI) based on the clinical presentation, specific electrocardiogram (ECG) alterations and serum cardiac enzyme levels. In the present study, data from patients with non-ST elevation AMI and ST elevation AMI were grouped together as AMI, and UA and AMI were grouped as acute coronary syndrome (ACS). Exclusion criteria included: normal or minimally diseased coronary angiogram, documented or inducible coronary artery spasm, valvular heart disease, current infection, drug abuse and severe hematological diseases such as leukemia. Patients treated with thrombolytics were also excluded. 392 subjects were finally enrolled in this study and consisted of 169 SA, 153 UA and 70 AMI subjects. A detailed medical history was obtained for each participant either from clinical records.

\subsection{Blood sampling and preparation}

All laboratory parameters were determined in the central laboratory of the Gangnam Severance hospital. Routine venipuncture was done at antecubital vein after an overnight fast. Serum glucose, total cholesterol, triglyceride, and high-density lipoprotein (HDL)-cholesterol, low-density lipoprotein (LDL)cholesterol, blood urea nitrogen (BUN), creatinine, total protein, albumin levels were measured via enzymatic procedures using a chemistry analyzer (ADVIA 1650, Siemens Healthcare, Tarrytown, NY, USA). High-sensitivity C-reactive protein (hs-CRP) was measured using a latex-enhanced immunoturbidimetric assay in an ADVIA 1650 (Siemens Healthcare, Tarrytown, NY, USA). Complete blood count (CBC) was measured using ADVIA 2120i (Siemens Healthcare, Tarrytown, NY, USA). 


\subsection{Measurements of hemorheologic parameters}

Blood samples for hemorheologic parameters were obtained via the vascular sheath into commercial EDTA $(1.5 \mathrm{mg} / \mathrm{ml})$ tube just prior to angiography. All measurements were complied following the new guidelines for hemorheological measurements provided by ISCH [1]. All hemorheological parameters were measured with a microfluidic hemorheometer (Rheoscan-AnD300; Rheo-Meditech, Seoul, Korea).

Additionally, determination of whole blood viscosity (WBV) was conducted in a research manner. However, the used test flow cells (K-03, Rheomeditech., Seoul, Korea) consisted of two chambers and a capillary tube which dimensions were $0.5 \mathrm{~mm}$ in diameter and $50 \mathrm{~mm}$ in length, respectively. Blood viscosity was calculated by processing the time-varying pressure data and calculated results were adjusted using Casson's equation.

The Critical shear stress (CSS) was measured using a native whole blood without adjusting hematocrit. The critical shear is defined as a minimum shear-stress that is required to disperse RBC aggregates [18]. For the CSS measurement, a transient microfluidic technique was adopted with optical detection. When a whole blood sample stored in a reservoir chamber was driven by a pressure differential through a narrow micro-channel (K-01, Rheomeditech, Seoul, Korea), the pressure differential exponentially decreased with time and the flow ceased asymptotically. During the process, the time-varying backscattered light intensity and pressure data were recorded in a computer data file and analyzed. When the backscattered light yielded a maximum, the corresponding time and shear stress were determined as critical time and critical shear stress, respectively. Further details of this technique are provided elsewhere [18].

In addition, erythrocyte sedimentation rate (ESR) as a representative of RBC aggregation and inflammation. Each RBC-plasma sample was evaluated using standard, $200 \mathrm{~mm}$ tall Westergren tubes and determining the position of the RBC-plasma interface at 60 minutes.

\subsection{Statistical analyses}

All analyses were performed using SPSS for Windows (ver 18.0; SPSS Inc., Chicago, IL). Mean values of clinical characteristics were shown according as clinical diagnosis. All numerical data are presented as mean value \pm standard deviation and categorical variables were presented as frequencies and percentages. The difference between means was tested for significance using Student's $t$-test and one-way ANOVA; for proportions we used the $\chi^{2}$ test. Pearson's correlation analysis was performed to evaluate the relationships between clinical and CSS. A $p$ value less than 0.05 was considered statistically significant.

\section{Results}

\subsection{Population characteristics}

Demographic characteristics and clinical laboratory data for the study population are shown in Table 1. The BMI, the incidence of controlled hypertension, diabetes, dyslipidemia and smoking habit were not significantly different among the groups. But, number of male gender was greater and a little younger age revealed in acute coronary syndrome, especially in AMI group. Compared to the stable angina group, laboratory results revealed elevated systemic inflammatory markers in ACS: (1) White blood cell counts (WBC) were 19.4\% higher in AMI subjects ( $p<0.001)$; (2) hs-CRP values were 2.6-fold greater in the UA and 3.7-fold higher in the AMI group (Table 1). Blood urea nitrogen and creatinine values 
Table 1

Clinical characteristics according as clinical diagnosis

\begin{tabular}{|c|c|c|c|c|}
\hline & SA $(n=169)$ & $\mathrm{UA}(n=153)$ & $\operatorname{AMI}(n=70)$ & $p$ \\
\hline Age (years) & $63.7 \pm 8.7$ & $59.9 \pm 11.2$ & $59.0 \pm 14.0$ & 0.001 \\
\hline Gender (Male:Female) & $106: 63$ & $96: 57$ & $64: 6$ & $<0.001$ \\
\hline $\operatorname{BMI}\left(\mathrm{kg} / \mathrm{m}^{2}\right)$ & $24.2 \pm 3.8$ & $25.2 \pm 5.8$ & $25.7 \pm 5.3$ & 0.056 \\
\hline $\mathrm{WBC}\left(\times 10^{3} / \mu \mathrm{L}\right)$ & $7.2 \pm 4.3$ & $7.2 \pm 2.5$ & $8.6 \pm 2.9$ & 0.008 \\
\hline Hemoglobin (g/dL) & $13.3 \pm 3.0$ & $13.4 \pm 1.7$ & $13.6 \pm 2.0$ & 0.643 \\
\hline Hematocrit (\%) & $39.8 \pm 9.8$ & $40.2 \pm 5.6$ & $40.8 \pm 7.2$ & 0.578 \\
\hline FBS (mg/dL) & $98.7 \pm 15.3$ & $101.2 \pm 21.4$ & $103.4 \pm 19.7$ & 0.125 \\
\hline Total protein $(\mathrm{g} / \mathrm{dL})$ & $6.6 \pm 0.6$ & $6.6 \pm 0.6$ & $6.8 \pm 0.6$ & 0.078 \\
\hline Albumin (g/dL) & $4.3 \pm 0.5$ & $4.3 \pm 0.4$ & $4.4 \pm 0.4$ & 0.537 \\
\hline BUN (mg/dL) & $17.2 \pm 9.0$ & $14.7 \pm 4.8$ & $17.6 \pm 9.3$ & 0.004 \\
\hline $\mathrm{Cr}(\mathrm{mg} / \mathrm{dL})$ & $1.1 \pm 0.8$ & $0.9 \pm 0.2$ & $1.0 \pm 0.3$ & 0.035 \\
\hline hs-CRP (mg/L) & $3.4 \pm 8.9$ & $8.9 \pm 31.9$ & $12.6 \pm 24.9$ & 0.022 \\
\hline Fibrinogen (mg/dL) & $302.6 \pm 71.6$ & $317.2 \pm 78.1$ & $337.3 \pm 87.9$ & 0.031 \\
\hline Total cholesterol (mg/dL) & $162.4 \pm 39.7$ & $170.7 \pm 41.0$ & $167.8 \pm 49.1$ & 0.208 \\
\hline HDL-cholesterol (mg/dL) & $42.4 \pm 10.8$ & $43.6 \pm 15.2$ & $40.6 \pm 10.0$ & 0.285 \\
\hline Triglyceride (mg/dL) & $148.8 \pm 105.6$ & $136.3 \pm 87.6$ & $122.1 \pm 56.9$ & 0.127 \\
\hline LDL-cholesterol (mg/dL) & $94.2 \pm 30.4$ & $101.6 \pm 32.8$ & $99.3 \pm 37.1$ & 0.132 \\
\hline Hypertension, $n(\%)$ & $124(73.4)$ & $105(68.6)$ & $41(58.6)$ & 0.155 \\
\hline Diabetes, $n(\%)$ & $47(27.8)$ & $45(29.4)$ & $22(31.4)$ & 0.849 \\
\hline Current smoker, $n(\%)$ & 45 (26.6) & $51(33.3)$ & $26(37.1)$ & 0.425 \\
\hline Dyslipidemia, $n(\%)$ & $71(42.0)$ & $54(35.3)$ & $20(28.6)$ & 0.128 \\
\hline Family history of early CVD, $n(\%)$ & $32(18.9)$ & $31(20.3)$ & $21(30.0)$ & 0.039 \\
\hline
\end{tabular}

were significantly lower in UA group, and cholesterol profiles were not significantly different between groups.

\subsection{Hemorheologic parameters}

Whole blood viscosity under shear rate profiles for the patient groups are shown in Table 2. For data obtained at native hematocrit, viscosity profiles revealed higher in UA than SA, and higher in AMI than UA along at all shear rates. So, WBV is much higher in ACS than SA.

CSS, in unit of $\mathrm{mPa}$, was $25.7 \%$ higher in ACS $(333.8 \pm 147.8)$ than in SA $(265.4 \pm 149.9)(p<0.001)$. Interestingly, CSS between UA and AMI were not significantly different.

Mean value of ESR in patients groups were not significantly different, but showed increasing tendency having the rank order of AMI $>\mathrm{UA}>\mathrm{SA}$. The wide range of standard deviation of each group is the reason why the differences between groups could not be made.

\subsection{Correlation with critical shear stress with clinical laboratory data}

CSS was analyzed the correlation with all available clinical laboratory variables. CSS was highly correlated with inflammatory biomarkers, such as, WBC, hs-CRP, and the parameters related RBC aggregation, 
Table 2

Hemorheologic parameters according as clinical diagnosis

\begin{tabular}{lcccc}
\hline & SA $(n=169)$ & UA $(n=153)$ & AMI $(n=70)$ & $p$ \\
\hline $\begin{array}{l}\text { Whole blood viscosity (mPa s) } \\
\text { (under each shear rate, 1/s) }\end{array}$ & & & & \\
1 & $35.4 \pm 9.3$ & $36.4 \pm 10.6$ & $43.9 \pm 20.2$ & $<0.001$ \\
2 & $24.1 \pm 5.6$ & $24.8 \pm 6.4$ & $29.5 \pm 12.6$ & $<0.001$ \\
5 & $15.5 \pm 3.0$ & $15.9 \pm 3.4$ & $18.6 \pm 7.1$ & $<0.001$ \\
10 & $11.6 \pm 1.9$ & $11.9 \pm 2.2$ & $13.8 \pm 4.9$ & $<0.001$ \\
20 & $9.1 \pm 1.3$ & $9.3 \pm 1.5$ & $10.6 \pm 3.4$ & $<0.001$ \\
50 & $6.9 \pm 0.9$ & $7.0 \pm 1.1$ & $8.0 \pm 2.3$ & $<0.001$ \\
100 & $5.8 \pm 0.7$ & $5.9 \pm 0.9$ & $6.6 \pm 1.8$ & $<0.001$ \\
200 & $5.0 \pm 0.6$ & $5.1 \pm 0.8$ & $5.7 \pm 1.5$ & $<0.001$ \\
500 & $4.3 \pm 0.5$ & $4.4 \pm 0.7$ & $4.9 \pm 1.2$ & $<0.001$ \\
1000 & $3.9 \pm 0.5$ & $4.0 \pm 0.7$ & $4.4 \pm 1.1$ & $<0.001$ \\
Critical shear stress (mPa) & $265.4 \pm 149.9$ & $338.3 \pm 125.2$ & $324.0 \pm 155.5$ & $<0.001$ \\
ESR(mm/hr) & $12.4 \pm 14.2$ & $13.7 \pm 14.2$ & $15.7 \pm 16.2$ & 0.407 \\
\hline
\end{tabular}

SA: stable angina, UA: unstable angina, AMI: acute myocardial infarction, ESR: erythrocyte sedimentation rate.

Table 3

Pearson's correlation between critical shear stress and biochemical parameters

\begin{tabular}{lrr}
\hline & \multicolumn{2}{c}{ Critical shear stress } \\
\cline { 2 - 3 } & \multicolumn{1}{c}{$r$} & $p$ \\
\hline WBC & 0.271 & $<0.001$ \\
Hemoglobin & -0.113 & 0.013 \\
Hematocrit & -0.109 & 0.017 \\
Total protein & 0.002 & 0.967 \\
Albumin & -0.265 & $<0.001$ \\
BUN & -0.018 & 0.079 \\
Creatinine & 0.038 & 0.400 \\
Total cholesterol & 0.042 & 0.357 \\
HDL-cholesterol & -0.137 & 0.003 \\
Triglyceride & 0.042 & 0.367 \\
LDL-cholesterol & 0.065 & 0.169 \\
Fibrinogen & 0.572 & $<0.001$ \\
hs-CRP & 0.311 & $<0.001$ \\
ESR & 0.522 & $<0.001$ \\
\hline
\end{tabular}

BUN: blood urea nitrogen, HDL: high density lipoprotein, LDL: low density lipoprotein, ESR: erythrocyte sedimentation rate. 

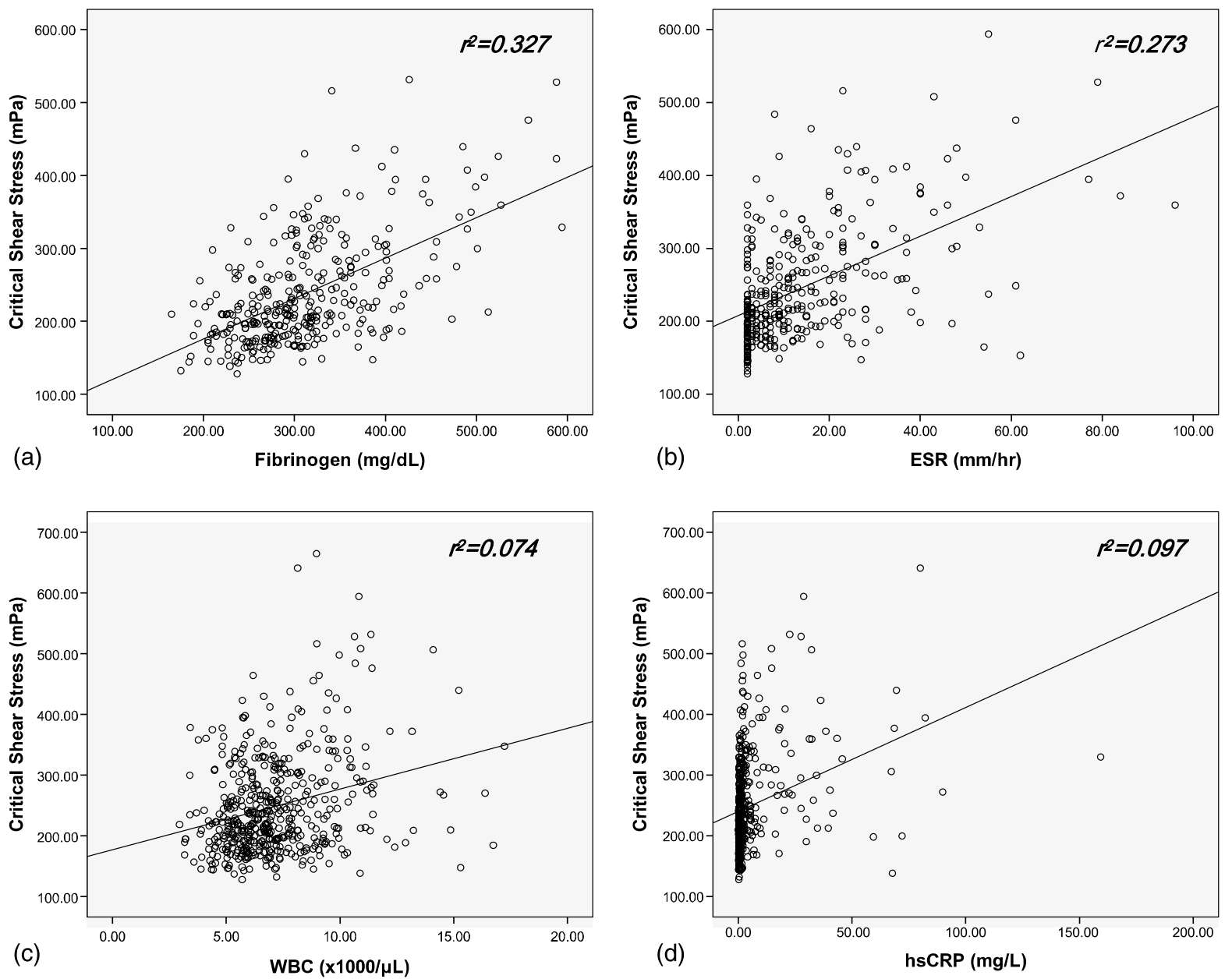

Fig. 1. Stronger correlations are shown between critical shear stress (CSS) and fibrinogen (a), and erythrocyte sedimentation rate (ESR) (b), than white blood cell count (WBC) (c), or highly sensitive C-reactive protein (hsCRP) (d).

such as, fibrinogen, ESR (Table 3). Hemoglobin and hematocrit were shown to be modest correlation with CSS. Among those variables, fibrinogen, and ESR were strongly correlated with CSS (Fig. 1).

\section{Discussion}

Hemorheologic alteration in vascular blood flow may lead to increase wall shear stress at the vascular endothelium $[5,12,13]$, thereby promoting occlusive thrombus formation in ACS. In addition, acute alterations of hemorheological parameters could serve as a trigger for the rupture of a vulnerable atherosclerotic plaque, thus aggravating thrombus formation and the clinical symptoms of ACS $[6,10]$. Among many hemorheologic parameters, the parameters associated with ACS are related to cell aggregation and blood viscosity which make high shear stress $[10,15]$. Also, it was reported that increased whole blood viscosity, altered RBC deformability and aggregability affect to the platelet reactivity on 
the anti-platelet therapy in acute coronary syndrome [4], and are related to the long term prognosis of transmural myocardial infarction in terms of inflammation [16, 17].

There are several indices for measuring $\mathrm{RBC}$ aggregation, such as, $\mathrm{M}$ and $\mathrm{M}_{1}$ from Myrenne Aggregometer (Myrenne GmbH, Roetgen, Germany) [8], amplitude (AMP), aggregation half time $\left(\mathrm{T}_{1 / 2}\right)$, surface area (SA), and aggregation index (AI) from LORCA - Laser-Assisted Optical Rotational Cell Analyzer (RR Mechatronics, Hoorn, The Netherlands) and RheoScan (AnD300, Rheomeditech, Seoul, Korea). Erythrocyte sedimentation rate (ESR) and low-shear blood viscosity are also known to reflect the extent of red cell aggregation [2,7].

Compared to other devices, the RheoScan-AnD300 (Rheomeditech, Seoul, Korea) might be useful in clinical field, because this device uses a disposable microchip with a disc-shaped test chamber for the RBC suspension being tested, and can get the results within 3 minutes. Furthermore, critical shear stress (CSS) by simultaneous measurement of shear stress and light backscattering using this device is very useful index of RBC aggregability [18]. We hypothesized that higher value of CSS might be associated with the patients with ACS. This study was objected to investigate CSS and other hemorheologic parameters in ACS and to evaluate the correlation with usual biomarkers for atherosclerosis such as fibrinogen, hs-CRP, and ESR.

The results of this study strongly support the hypothesis of a linkage between ACS and RBC aggregation indices, such as CSS, ESR, and low shear viscosity. And, those aggregation indices are strongly correlated with widely accepted risk factors for coronary artery disease, such as WBC, fibrinogen, as shown in Table 3 and Fig. 1. The hs-CRP of ACS was significantly elevated than SA. However, the correlation between hsCRP and CSS was not so strong. Compared to SA, CSS of ACS were significantly elevated. Interestingly, CSS of UA and AMI were not significantly different. This kind of phenomenon was also shown in other study [10], and it might be partially explained by that CSS is strongly dependent on RBC deformability [18] as well as fibrinogen concentration, which did not show significant difference between UA and AMI. However, we suspected that increased CSS might trigger the rupture of atherosclerotic plaque. Higher shear stress would be needed for overcoming CSS. While CSS is an index of red cell aggregation, it might affect the vascular endothelium by increasing shear force. Finally, WBV values showed much higher value in ACS patients. WBV in ACS is usually elevated. Thus, we may suggest that higher WBV and higher RBC aggregability produce increased wall shear stress, and thereby, trigger the ACS.

Hemorheologic properties, including RBC aggregation is known to be influenced by plasma macroptoteins and erythrocyte cell membrane properties. This properties might be affected by inflammatory cytokines and proteins produced in acute coronary syndrome. So, hemorheologic parameters and the clinical situation of acute coronary syndrome might affect mutually to each other [7, 10,17].

In summary, CSS which can be directly measured by the present transient, microfluidic aggregometer, were higher value in ACS than SA. CSS might provide the useful information on the characteristics of $\mathrm{RBC}$ aggregation-disaggregation and hemorheological risk for CAD. The CSS may be used in clinical field as an easily measurable novel risk marker for ACS. Further study with serial measurements of CSS in this patients group during clinical follow up, and prospective cohort study in normal population can clarify the long term role of CSS, as triggering parameters of ACS.

\section{Acknowledgments}

This work was partly supported the Seoul R\&BD Program Grant (NT080573), and by the R\&D program of MKE/KEIT (KI001810030039, Clinical Validation for Intravascular Therapeutic microrobot 
system), Republic of Korea. The authors comply with the Ethical Guidelines for Publication in Clinical Hemorheology and Microcirculation as published on the IOS Press website and in Volume 44, 2010, pp. 1-2 of this journal.

\section{References}

[1] O.K. Baskurt, M. Boynard, G. Cokelet, P. Connes, B. Cooke, S. Forconi, F. Liao, M. Hardeman, F. Jung, H. Meiselman, G. Nash, N. Nemeth, B. Neu, B. Sandhagen, S. Shin, G. Thurston and J. Wautier, New guidelines for hemorheological laboratory techniques, Clinic Hemorheol Microcirc 42 (2009), 75-97.

[2] O.K. Baskurt, M. Uyuklu, P. Ulker, M. Cengiz, N. Nemeth, T. Alexy, S. Shin, M.R. Hardeman and H.J. Meiselman, Comparison of three instruments for measuring red blood cell aggregation, Clin Hemorheol Microcirc 43 (2009), 283298.

[3] N. Beamer, G. Giraud, W. Clark, M. Wynn and B. Coull, Diabetes, hypertension and erythrocyte aggregation in acute stroke, Cerebrovascular Diseases 7 (1997), 144-149.

[4] E. Cecchi, R. Marcucci, R. Paniccia, B. Bandinelli, S. Valente, C. Giglioli, C. Lazzeri, G.F. Gensini, R. Abbate and L. Mannini, Effect of blood hematocrit and erythrocyte deformability on adenosine 5'-diphosphate platelet reactivity in patients with acute coronary syndromes on dual antiplatelet therapy, Am J Cardiol 104 (2009), 764-768.

[5] G.R. Cokelet, The rheology of human blood, in: Biomechanics, Y.C. Fung, N. Perrone and M. Anliker, eds, Prentice-Hall, Englewood Cliffs, 1972, pp. 63-103.

[6] R. Corti, V. Fuster and J.J. Badimon, Pathogenetic concepts of acute coronary syndromes, J Am Coll Cardiol 41 (2003), 7S-14S.

[7] M.R. Hardeman, P.T. Goedhart and S. Shin, Methods in hemorheology, in: Handbook Hemorheology and Hemodynamics, O.K. Baskurt, M.R. Hardeman, M.W. Rampling and H.J. Meiselman, eds, IOS Press, Amsterdam, 2007, pp. 242-266.

[8] H. Kiesewetter, H. Radtke, R. Schneider, K. Mussler, A. Scheffler and H. Schmid-Schonbein, The mini erythrocyte aggregometer: A new apparatus for the rapid quantification of the extent of erythrocyte aggregation, Biomed Tech (Berlin) 28 (1982), 209-213.

[9] R.A. Kloner, Can we trigger an acute coronary syndrome? Heart 92 (2006), 1009-1010.

[10] B. Lee, A. Durairaj, A. Mehra, R. Wenby, H. Meiselman and T. Alexy, Hemorheological abnormalities in stable angina and acute coronary syndromes, Clin Hemorheol Microcirc 39 (2008), 43-51.

[11] H. Lim, Y. Lee, J. Nam, S. Chung and S. Shin, Temperature-dependent threshold shear stress of red blood cell aggregation, Journal of Biomechanics 43 (2010), 546-550.

[12] M. Naghavi, P. Libby, E. Falk, S.W. Casscells, S. Litovsky, J. Rumberger, J.J. Badimon, C. Stefanadis, P. Moreno, G. Pasterkamp, Z. Fayad, P.H. Stone, S. Waxman, P. Raggi, M. Madjid, A. Zarrabi, A. Burke, C. Yuan, P.J. Fitzgerald, D.S. Siscovick, C.L. de Korte, M. Aikawa, K.E. Airaksinen, G. Assmann, C.R. Becker, J.H. Chesebro, A. Farb, Z.S. Galis, C. Jackson, I.K. Jang, W. Koenig, R.A. Lodder, K. March, J. Demirovic, M. Navab, S.G. Priori, M.D. Rekhter, R. Bahr, S.M. Grundy, R. Mehran, A. Colombo, E. Boerwinkle, C. Ballantyne, W. Insull Jr, R.S. Schwartz, R. Vogel, P.W. Serruys, G.K. Hansson, D.P. Faxon, S. Kaul, H. Drexler, P. Greenland, J.E. Muller, R. Virmani, P.M. Ridker, D.P. Zipes, P.K. Shah and J.T. Willerson, From vulnerable plaque to vulnerable patient: A call for new definitions and risk assessment strategies: Part I, Circulation 108 (2003), 1664-1672.

[13] M. Naghavi, P. Libby, E. Falk, S.W. Casscells, S. Litovsky, J. Rumberger, J.J. Badimon, C. Stefanadis, P. Moreno, G. Pasterkamp, Z. Fayad, P.H. Stone, S. Waxman, P. Raggi, M. Madjid, A. Zarrabi, A. Burke, C. Yuan, P.J. Fitzgerald, D.S. Siscovick, C.L. de Korte, M. Aikawa, K.E. Airaksinen, G. Assmann, C.R. Becker, J.H. Chesebro, A. Farb, Z.S. Galis, C. Jackson, I.K. Jang, W. Koenig, R.A. Lodder, K. March, J. Demirovic, M. Navab, S.G. Priori, M.D. Rekhter, R. Bahr, S.M. Grundy, R. Mehran, A. Colombo, E. Boerwinkle, C. Ballantyne, W. Insull Jr, R.S. Schwartz, R. Vogel, P.W. Serruys, G.K. Hansson, D.P. Faxon, S. Kaul, H. Drexler, P. Greenland, J.E. Muller, R. Virmani, P.M. Ridker, D.P. Zipes, P.K. Shah and J.T. Willerson, From vulnerable plaque to vulnerable patient: A call for new definitions and risk assessment strategies: Part II, Circulation 108 (2003), 1772-1778.

[14] T. Ohtani, Y. Ueda, I. Mizote, J. Oyabu, K. Okada, A. Hirayama and K. Kodama, Number of yellow plaques detected in a coronary artery is associated with future risk of acute coronary syndrome: Detection of vulnerable patients by angioscopy, J Am Coll Cardiol 19 (2006), 2194-2200.

[15] U. Rauch, J.I. Osende, V. Fuster, J.J. Badimon, Z. Fayad and J.H. Chesebro, Thrombus formation on atheroscleroticplaques: Pathogenesis and clinical consequences, Ann Intern Med 134 (2001), 224-238. 
[16] L. Sargento, H.S. Do Rosário, C. Perdigão, J. Monteiro, C. Saldanha and J.M.E. Silva, Long-term prognostic value of the hemorheological profile in transmural myocardial infarction survivors: 60-month clinical follow-up, Rev Port Cardiol 21 (2002), 1263-1275.

[17] L. Sargento, C. Saldanha, J. Monteiro, C. Perdigão and J.M.E. Silva, Long-term prognostic value of protein C activity, erythrocyte aggregation and membrane fluidity in transmural myocardial infarction, Thromb Haemost 94 (2005), 380-388.

[18] S. Shin, J. Nam, J. Hou and J. Suh, A transient, microfluidic approach to the investigation of erythrocyte aggregation: The threshold shear-stress for erythrocyte disaggregation, Clin Hemorheol Microcirc 42 (2009), 117-125.

[19] Y.T. Yaylali, I. Susam, E. Demir, M. Bor-Kucukatay, B. Uludag, E. Kilic-Toprak, G. Erken and D. Dursunoglu, Increased red blood cell deformability and decreased aggregation as potential adaptive mechanisms in the slow coronary flow phenomenon, Coron Artery Dis 24 (2013), 11-15.

[20] F. Yu, J. Armstrong, J. Tripette, H. Meiselman and G. Cloutier, A local increase in red blood cell aggregation can trigger deep vein thrombosis: Evidence based on quantitative cellular ultrasound imaging, Journal of Thrombosis and Haemostasis 9 (2011), 481-488. 